\title{
Selecting and Training Students with No Suitable Informatics Background for Informatics Olympiads - The Case of Syrian Olympiad in Informatics
}

\author{
Ammar ALNAHHAS ${ }^{1}$, Emad ALAZAB ${ }^{2}$ \\ ${ }^{1}$ Damascus University, Syria \\ ${ }^{2}$ National Commission for the Syrian Science Olympiad, Syria \\ e-mail:eng.a.alnahhas@gmail.com,emadalazab@gmail.com
}

\begin{abstract}
Many countries suffer from a bad informatics syllabus in their educational systems, which leaves the students with no skills in computer programming or algorithms. This fact imposes a big challenge to the process of selecting the best secondary school students for the informatics Olympiad; it also makes training them a harder process.

In this paper, we present our vision to tackle this issue in Syria, where the educational system lacks the principles of computer algorithms; moreover, the educational system tends to target student skills that do not include creativity nor innovation. We show the process of gradual selection of students along with the scientific materials of contests in each step, we present the training process in all stages as well. As the process evolved in the last ten years, the statistics of the enrolled students as well as the practical results were provided at last.
\end{abstract}

Keywords: informatics olympiad, Syria, Syrian olympiad in informatics, programming contest.

\section{Introduction}

It is now 11 years since the first Syrian Olympiad in Informatics was held. Syria started participating in the International Olympiad in informatics in 2004, it has not been easy for us in Syria to organize a local competition. It was still very difficult for us to train students that are going to participate in IOI; this is mainly because of many reasons, which vary from social, educational and logistical ones.

Syrian universities had no computer science major before 2001, moreover the school syllabus had no real informatics content before 2005; hence the informatics education was almost absent from the society. However, the informatics education has been improved significantly in the last decade; we have been suffering from many problems:

- School informatics syllabus focuses on computer usage principles, such as using the operating system, the internet and some famous applications; it has no serious content related to computer programming and algorithms. The informatics subject 
does not exist in the curriculum of general secondary education certificate, which dissuades students from learning informatics.

- Informatics schoolteachers are not qualified, professional teachers tend to not to teach at schools because of bad syllabus content, therefore anyone with an ICDL certificate is allowed to teach informatics in schools.

- General school educational concepts tend to make students memorize rather than think about problem solving, this makes students much more far from learning computer algorithms and programming and deepens the gap between students and informatics.

Because of the problems mentioned earlier selecting and training students for informatics Olympiad is a very tough mission, as it is hard to find suitable students, it is hard to convince them to participate, and it is hard to train them in a manner that they can challenge international students.

In the last ten years, we have tried to find solutions to these problems, by inventing selection and training models. At first we tried a model based on the concept of "Training first", while starting from 2012 we started a new model based on "selection first". In this paper we discuss the details of these two models, the first model is viewed in section 2, the second model is presented in section 3, section 4 shows the comparison between the two models, we conclude the paper in section 5.

\section{2. "Training First" Model}

When the first Syrian national Olympiad in informatics was held in 2004, it was very tough to find students that can be trained and selected for the IOI. So two strategies were introduced at that time: the first strategy was a "long term training" which is based on training young children so that they can be qualified to learn computer programming and algorithms after years of training, the second strategy is to select students that should be trained and prepared to participate in IOI in the same year.

To achieve the two mentioned strategies, students are classified into divisions according to their ages; the final distribution of divisions was to position students whose age is below 12 in the first division, students with age below 15 in the second division and students of the secondary school who were younger than 20 are considered in the third group. The training materials of the first and second groups were chosen to improve the mental capabilities of students, the content was about logic games, mathematics and simple algorithms, later "Scratch" programming language was used to train students in these two groups, details of using Scratch was published earlier (IDLBI, 2009).

The students of the third division were chosen and trained to participate in IOI, it is obvious that students of the second division were being moved automatically when they became older to be members of the third division so they can participate in IOI. But here we faced a problem that the number of students in the second division who are eligible to move to the third division was very low, which makes it hard to create a challenging environment for students, and the candidates of Syrian national team was very limited. Therefore, we had to start a new selection and training process with three stages: 
selection, training and qualification, whereas another process of preparing students for national competition was held, the winners of the national competitions are prepared to participate in the next year's IOI.

\subsection{Selecting Students}

The first stage of selecting students is held in each Syrian province separately, each province has a separate team who is responsible for selecting and training their students.

The selection process is based on IQ tests, because at that point it is impossible to test students' abilities in computer programming and algorithms, since they lack the minimum amount of knowledge to test their abilities. The aim of these IQ tests is to find the best students that are suitable to be taught IOI materials, we believed that we should look for students that had talent, creativity, intelligence and a quick-wit.

The first problem we faced here is the general society view of the informatics Olympiad, the school teachers have no background nor are interested to know, that is because of the large gap between the school informatics definition and the one intended in IOI. The solution was to hold the selective IQ tests centrally by the organizers of the national informatics Olympiad.

The first problem causes another problem of organizing a selection test that should accept a huge number of students, we insisted on making this selection on site test so that we can ensure the up most integrity.

This selection process was just elementary we wanted to choose about approximately 1000 students that would be trained and then the last teams would be selected from this student group.

\subsection{Training Stage}

This is the toughest part; we had to teach students the principles of computer programming and algorithms in about eight months.

As we had selected students with high IQ we expected that they were able to learn algorithms and programming easier and faster than usual people.

The selected students had to attend two concurrent courses: one for computer programming and one for computer algorithms. The programming course was based on learning "Scratch" programming language which was a good starting point into programming, the rest of the course focuses on real programming languages concepts. We noticed that there was a general acceptance of learning programming; the students who were not able to adapt to the materials left the course, so it was a process of natural selection along with the students' education. The algorithm course focuses on the principles of simple computer algorithms, we could not present advanced algorithms concepts as the experience in computer algorithms can be gained by practicing and solving problems rather than just getting theoretical knowledge, which will not be useful until it is applied in a real experience. 
The training process of this stage was not that useful because of the following reasons:

- The training was held in 14 provinces of Syria where each province training team is responsible for training their own students, the training teams were not fully qualified, and differ in experience.

- The number of students was very large for the trainer to follow up with all of them, so that the theoretical knowledge was not backed by any practical training, this caused a loss of the efforts of trainers.

We tried to solve the first problem by conducting a training camp for trainers, the goal of these camps was to try to improve the trainers' experience and direct their efforts in the correct direction, these camps proved their effectiveness for first and second division group. However, the third group trainers were not benefiting through them, as gaining the experience to train computer algorithms needs a long time practicing which cannot be gained in short term camps.

The second problem was not solvable in this training model and was one of the reasons to move to a new model, which is going to be presented later in this paper.

At the end of the training two groups of students were chosen for each province, the first group is chosen to proceed to the IOI team selection, where the other group is chosen to participate in the national Olympiad as the team of this province.

\subsection{Selection}

The IOI team selection was based on series of contests, the selection is based on the average of these contests, the students allowed to participate in these contests are the groups of students selected by each province training team, along with the winners of the past national Olympiad, the team members of the last years are also allowed to participate.

A special training program was prepared for the selected team, training camps were also held to support students in the team.

\subsection{This Model in Brief}

The model we had used for five year (from 2006 to 2011) was based on selecting an initial group of possible suitable students, training all of them for the whole year then choosing the teams. The team members still participated in the selection competitions for the next years if they still meet the age of IOI. The winners of the national competition were also allowed to participate in the next year's selection competitions. Fig. 1 visualize this process.

This model has many drawbacks; the selection process does not guarantee attracting suitable students. The training process does not cover the most important practical part, and the most important is that the whole system does not create the motive for the 


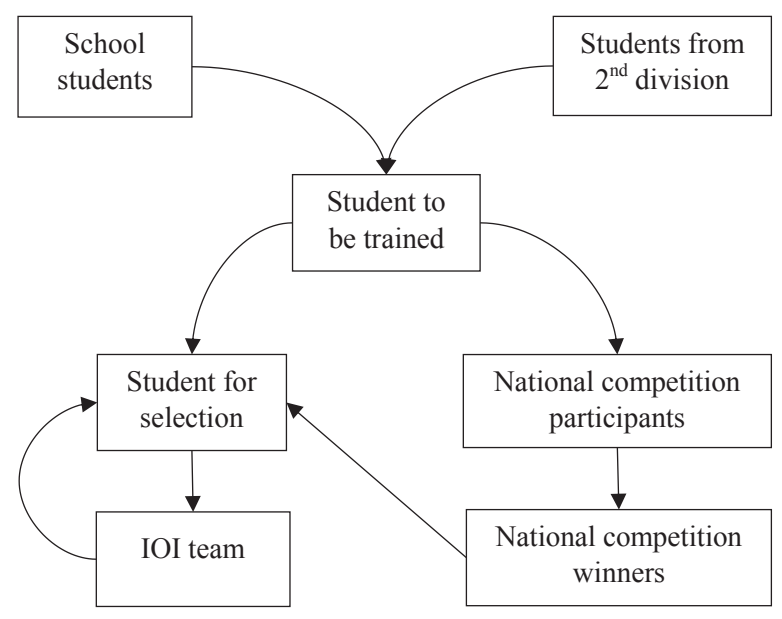

Fig. 1. the "training first" model.

students to start training by themselves, trying to improve their own problem solving skills, which needs a long time of practice, and is important for any novice programmer (Lahtinen et al., 2005).

\section{3. "Selection First" Model}

This new model was adopted in 2012, the main goal of this model is to select students first then concentrate on training the selected small group of students, we thought that enhancing the selection process and making it more effective would help us increase the ratio of suitable students as a result of the selection.

We had to build the selection process to avoid selecting students according to their school marks, which is not suitable for evaluation in this case. The student's experience in computer programming is useless for the evaluation as well, as they have no previous knowledge because of syllabus issues as mentioned in the introduction. Moreover, the only way to reach the maximum number of students is to reach their schools, unfortunately, we cannot rely on schoolteachers to help us choose students as teachers are not qualified.

We decided to start from schools, we had to reach the maximum number of students and we cannot conduct an online contest, because the students will have no motive to participate and we wanted to build a general society acceptance of the Olympiad as a good activity for students. Besides, the integrity of online contests is not guaranteed and proctoring about 5000 students is not possible at all.

Each school selects its team, then teams from the same area participate in a competition to select the area team, the area teams participate in a competition to select the province team and the teams of provinces participate in the final national competition. 


\subsection{Teachers' Olympiad}

As mentioned earlier the starting point is the schools, and as the teachers of school are in charge of selecting students in this important stage, we had to qualify and train teachers so they can help us choose the most suitable students.

The teachers' Olympiad is a competition that informatics schoolteachers can participate in. The completion tasks are similar to those presented to students during selection process. The goal of this completion is to achieve the following:

- Spread the informatics Olympiad among teachers, school administrators and the whole society.

- Let the teachers be introduced to what informatics Olympiad is about.

- Get good statistics about teachers' qualifications and find the good teachers who can be trained and prepared to be a trainer.

Teachers of informatics in Syrian schools are not specialists; they have a good knowledge in computer general information, computer usage such as using the operating system and applications, some teachers know the principles of computer programming but they constitute no more than $5 \%$ of total number of teachers. Hence, we faced a new challenge, which is inventing tasks that can be solvable by teachers as well as targeting the goal that the competition is all about.

The tasks used in the teachers' competition are based on computer algorithms without programming, that is, the solution of the questions is based on finding the correct algorithm without the need to program it, these types of tasks are used later in the student selection process, but using them here has some advantages:

- The type of tasks is algorithmic, that reflects the correct image of informatics Olympiad.

- These tasks can help discover teachers with good ability to be prepared to train students later as it reflects their creativity, innovation and intelligence.

The competition had a good impact on participants, the type of tasks had a general acceptance among teachers, and as we observed it almost achieved the goal it was made for.

\subsection{Student Selection - School Phase}

To reach almost all secondary school students in the country, the selection process starts from the school itself. As there is still no general overview of informatics Olympiad in the society, starting from schools creates a good challenging atmosphere that help motivating students to participate.

Each school chooses its selection criteria, which depends usually on a quick IQ and math tests, the team of each school consists of five students that should participate in the next selection phase. 


\subsection{Student Selection - Area Phase}

The students of schools at the same area competes in the same site, at this moment we have students with no programming or algorithmic background, but they are chosen as the best of their colleagues. The target of this selection phase is to choose students that are eligible to go on, we believe that students with creativity, innovation and high ability to understand simple tasks and find a solution to sophisticated riddles are best suitable for being trained on computer programming and algorithms. IQ tests are a good measure for intelligence scores are closely associated with creativity scores for secondary school students (Kim, 2005). So the competition in this phase consists of a number of IQ questions, some of which are shaped like a real life problem that the student should investigate, analyze and find a good method to reach the solution.

Both students and teachers generally accepted this type of questions, it seemed familiar to the students, as it is not related to any school syllabus content, it also encouraged the qualified students to practice preparing for the next selection phase. Five students are chosen from each area to participate in the next phase.

\subsection{Student Selection - Province Phase}

In this selection phase, students still have no programming skills, but they are talented. We have to prepare tasks that are both solvable by students and are related to IOI content.

The solution was to prepare tasks that have an algorithmic nature, but students should solve them by hand, this can attract students that know nothing about programming (Marcin KUBICA, 2010) especially innovative students with good thinking abilities.

The tasks of this phase are chosen to meet the following requirements:

- It has the same programming problem structure (input, output).

- It requires some algorithm to be invented for the given test case be solvable by feasible time when solved by hand.

- It is not multiple-choice task; students should find the output.

The task statement is similar to programming tasks, the student is given one or two inputs, and he/she should find a good algorithm to get to the correct output, Table 1 shows some task samples

The tasks achieved many benefits to the students such as:

- Make the students familiar with programming problems.

- Teach students that finding the correct result as well as the algorithm is important.

- Select students that have talent and have a good algorithmic thinking and good problem solving abilities.

In addition to the mentioned type of tasks, another type is used to introduce students to algorithm analysis, this type of tasks presents an algorithm to students then asks some questions that encourages them to understand and analyze the algorithm. This type of 
Table 1

Sample tasks for province selection phase

Given a sequence of letters (ABACBCDBCD):

- Find the number of increasing subsequences.

- Find the longest increasing subsequence.

We have towers of coins where the number of coins in each tower is given: 610423

Find the minimum number of coins that should be moved to make the number of coins equal in all towers.

We want to buy 1000 liter of milk, there are 10 salesmen, and each has a quantity of milk and has a specific price, find the minimum money to be paid to buy the desired amount of milk.

tasks helps selecting students with good abilities in self-learning and problem solving; Table 2 shows a sample of this task.

Although the competition is held in all province centers at the same time; where each student compete in the province center he lives in, the result of all students is merged, then the best sixty students are chosen to move on to the national competition.

\subsection{National Competition}

The qualified students to the finals are trained for programming for the first time after the province selection phase. The students showed good and fast training skills, most of them accepted programming concepts easily, and they learned $\mathrm{C}++$ programming language principles and simple algorithm concepts in a small period.

Table 2

Sample of second type province selection tasks

Given the following grid of number where all rows and columns are sorted:

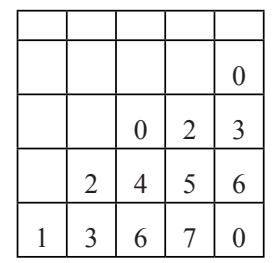

To search for a number in this grid we start from the square in the lower-left corner, if the number we are searching for is greater than the number in this square, we remove the first column of the grid, otherwise we remove the last row of grid, and we do the same method with the remaining grid.

- How many comparison operations are needed to find number 10 ?

- How many comparison operations are needed to make sure the number does not exists in the worst case?

- Answer the last question with another grid with $\mathrm{n}$ rows and $\mathrm{m}$ columns. 
The students attend an intensive training program in the period between province selection competition and national finals; training includes conducting lectures in training centers, online lectures, recorded sessions. Students are encouraged to learn by themselves, they are provided by books, introduced to online resources and online training websites. The usage of social networks to establish communication between students and trainers proved a good efficiency in improving student skills as they are motivated by teamwork and challenging other students, feeling in direct and continuous contact with trainer pushes them to train more and get help when needed. In usual people are interested in learning using social networking (Acharya et al., 2013).

The national competition is an IOI-like competition with algorithms and computer programming tasks, the system used in Syrian national Olympiad since 2012 is the wellknown CMS system (Maggiolo and Mascellani, 2012).

The best ten students wins the competition and join the so-called national team; which is the group of the winners of national competitions in last few years.

\subsection{IOI Team Selection}

The new students in the national team start training after the national competition, where old students have been training since they had joined the national team. All the national team students participate in a selection competition. The competition tasks are tough and are selected to be similar to IOI tasks level, the four winners of this competition are chosen to be the IOI team of Syria.

The IOI team selection is the last phase of the selection process of this model, Fig. 2 visualize the whole process.

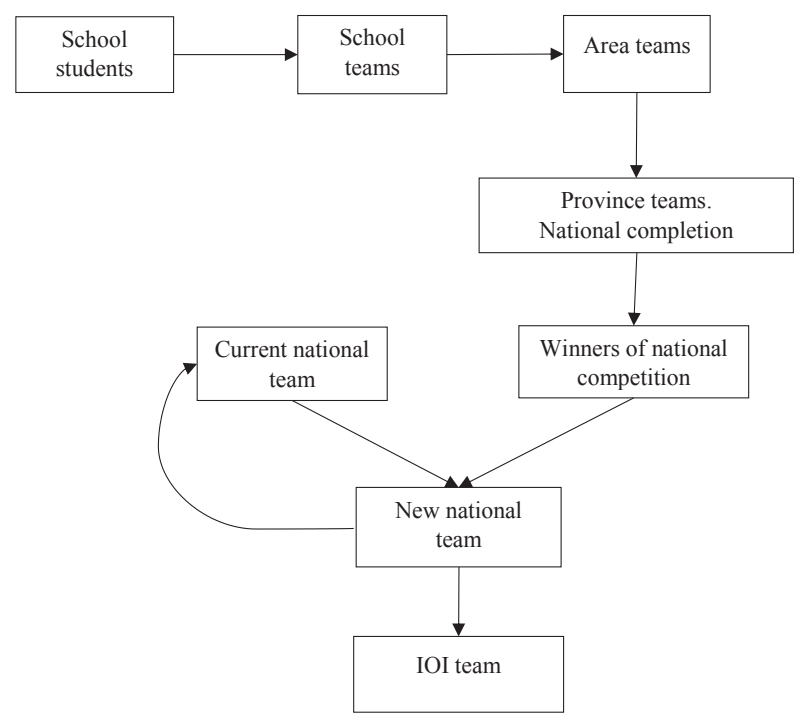

Fig. 2. The "Selection first" model. 


\subsection{Training}

Training is the most important part of the whole process, to achieve good results selected students should be trained properly.

The training is important in three periods:

Between province selection competition and national competition: in this phase the training starts by presenting basic programming and algorithms as described earlier in this paper.

Between the national competition and the IOI selection competition: In this period the training is based mainly on online lectures and online competitions, students are encouraged to solve as many problems as possible, they are followed up daily and directed to any resources and materials needed.

After the IOI team selection: The training continues for all students in the national team, the training is continuous and is based on the following:

- Online lectures that are provided via a special e-learning server.

- Training camps are conducted two times in the summer, where students meets for a month to training.

- Online competitions: Online competition has proved a high efficiency in training students, an online contest is prepared almost every week, which has a good acceptance among students, where they find it a good place to show their evolution and improvements. It provided a good challenging environment that motivate students to make advantages to beat each other; it also provides a good tool to introduce new ideas to students by presenting it inside problems. Moreover, when using problems from old competitions student can estimate their levels internationally and make more improvements. Our observations conforms to researches shows that online programming contests can be used to build programming trainings (Combéfis and Wautelet, 2014)

\section{Comparing "Training First" Model to "Selection First Model"}

The first model is based on training a large number of students, then selecting best students after training, this strategy did not suites the case of Syrian Olympiad in informatics because of many reasons including:

- Failing to attract many talented students, thus losing them in Olympiad.

- Large number of students makes training need a lot of stuff, resources and time, lacking professional people and needed resources makes this model not suitable.

- Training students with different abilities with the same training material is not suitable, good students may not benefit at all, where bad students fail in the selection.

This model was used for six years, the average participating students count at the first initial selection is 500 , which is relatively a small number, the average students participating in the final selection count is about 20 , the average non-zero marks rate in 
the last selection is $75 \%$, and the average non-zero marks rate in the national competition is $70 \%$.

The second model is used since 2012; it is based on choosing best students gradually using suitable selection criteria, the selected students are then trained and prepared for IOI participation.

The process of this model has proved good advantages, start selecting students from schools makes it possible to target almost all secondary school students, spreading out the culture of Olympiad among society. Providing students with algorithmic and programming content gradually has proved good results as well, focusing efforts to train small group of good students and improving the training process has a good impact on students in general.

Table 3 shows statistics of participating students since 2012, we consider non-zero mark in the final competition as a criterion to measure the success of student selection and training in the small period between province selection contest and final competition, the average non-zero marks count is $80 \%$.

The national team students prove good abilities in learning new concepts, training and competing. In the last four years, they started to have good ranks in famous online contests and training platforms, like Codeforces.

\section{Conclusion}

In this paper, we viewed the Syrian Olympiad in informatics experience in the last 11 years, we talked about the difficulties we faced, including poor informatics teaching and lack of professional trainers, and we presented two different models that were used in Syria. The first model depends on training a large number of students in order to select teams from them; this model proved that it is not that suitable. The second model is based on choosing good students according to a special criteria, then training them.

The feedback of the whole process is analyzed each year, and many improvements are planned to be added, merging the bright side of the first model with the second model is going to help improving our strategy in the next years, which will improve the student training process and make national informatics Olympiad a more successful story that will help improving the society.

Table 3

Student participation statistics

\begin{tabular}{lrrr}
\hline & 2012 & 2013 & 2014 \\
\hline Schools & 7550 & 7020 & 7980 \\
Areas & 2334 & 2940 & 3250 \\
Provinces & 356 & 542 & 620 \\
Finals & 62 & 51 & 72 \\
\hline
\end{tabular}




\section{References}

Acharya, V., Patel, A., Jethava, S. (2013). A survey on social networking to enhance the teaching and learning process. International Journal of Advanced Research in Computer Science and Software Engineering, $3(6)$.

Combéfis, S., Wautelet, J. (2014). Programming trainings and informatics teaching through online contests. Olympiads in Informatics, 8, 21-34.

Idlbi, A. (2009). Taking kids into programming (contests) with. Olympiads in Informatics, 3, 17-25.

Kim, K. H. (2005). Can only intelligent piople be creative? The Jouranl Of Secondary Gifted Education, XVI, 57-66.

Lahtinen, E., Ala-Mutka, K., Järvinen, H.-M. (2005). A study of the difficulties of novice programmers. In: Proceedings of the 10th Annual SIGCSE Conference on Innovation and Technology in Computer Science Education. New York, ACM SIGCSE Bulletin. 14-18

Maggiolo, S., Mascellani, G. (2012). Introducing CMS: a contest management system. Olympiads in Informatics, 6, 86-99.

Marcin KUBICA, J. R. (2010). Algorithms without programming. Olympiads in Informatics, 4, 52-66.

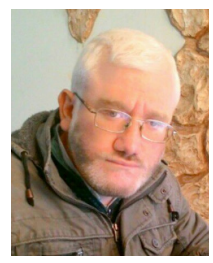

A. Alnahhas - Holds M.Sc. in Computer science from Damascus University and is preparing for $\mathrm{PhD}$, he was a former IOI contestant and has been involved in coordinating Syrian Olympiad in Informatics and training students since 2005, he has many contribution for the national Olympiad including preparing tasks and development grading systems.

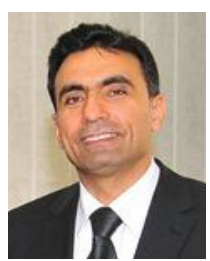

E. Alazab - The head of the National commission for the Syrian Science Olympiad. 